\title{
Classifying Iris Image based on Hierarchical Visual Codebook and Encryption using Bio-Chaotic Algorithm (BCA)
}

\author{
Rashmi M. Mhatre \\ ME-II Computer Engineering \\ G.H. Raisoni Institute of Engineering and \\ Technology \\ Pune, India
}

\author{
Deeksha Bhardwaj \\ Department of Computer Engineering \\ G.H. Raisoni Institute of Engineering and \\ Technology \\ Pune, India
}

\begin{abstract}
The classification of the iris image under the fake or real and also adding security to it by encrypting it provides double the security. Iris recognition system can undergo the security attacks which can result into the fraudulent identity authentication. The attacker therefore will try to develop the methods which will spoof the iris biometrics. Therefore it becomes difficult to develop the recognition system which will be attack proof. The one of the solution to this is iris liveness detection, where fake and the real iris images are classified and detected. As it is known that the anti-virus industry establishes the computer as well as internet virus databases to tackle the problem of viruses, malwares etc ,these database is dynamically get updated as they are share via public domain and can use this concept to tackle the fake iris images by preparing iris database. So, in this project will try to classify the iris images into the fake and real images, and store those into database along with this we are going to use the cryptographic algorithm to achieve the security. The use of the bio-chaotic stream cipher will help to encrypt the iris images and store them securely with the help of biometric key and bio-chaotic function.
\end{abstract}

\section{General Terms}

Pattern Recognition, Security, Bio-chaotic Algorithms.

\section{Keywords}

Iris image classification, Hierarchical Visual Codebook (HVC), iris liveness detection, race classification, coarse-tofine iris identification.

\section{INTRODUCTION}

IRIS recognition has become a great analysis topic due to its wide applications in security like banking, border management, national ID card, etc. Iris could be a annulate region of human eye with rich texture data close to infrared whet. Iris texture is considered associate degree epigenetic biometric pattern and stable throughout life in order that iris recognition provides associate degree extremely reliable methodology for individual authentication. Iris recognition aims to assign a singular identity label to each iris image supported automatic preprocessing, feature analysis and have matching progressive iris recognition ways embrace physicist section reception, ordinal measures.

In ancient iris recognition applications, iris pictures taken from somebody's eye are outlined because the same category in order that the dissimilarity between iris pictures of various subjects should be known. However, some applications in iris biometrics ought to realize the similarity between completely different subjects, that classify iris pictures into many specific classes. For instance, in iris physiological property detection, one has to classify all iris pictures into two classes, real or faux iris images; in some rhetorical or industrial applications, the racial data of iris pictures could also be needed, e.g. race classification of iris pictures into Asian and non-Asian subjects. Besides, the classification of all iris pictures within the central information into multiple classes could facilitate speed up large-scale iris identification. To satisfy the wants of these vital applications towards a secure, efficient and convenient society, iris image classification ways are necessary to assign an application specific category label (genuine vs. fake, Asian vs. non-Asian, etc.) to every iris image. Iris physiological property detection, race classification, and coarse-to-fine iris identification (or iris indexing) are typical applications of iris image classification, so that they will be unified into a general framework.

The Hierachical visual Codebook is a combination of two well known Bag-of-Words models method, namely Vocabulary Tree (VT), and Locality-constrained Linear Coding (LLC). The recent image classification systems includes two vital parts: bag-of-features (BoF) and spatial pyramid matching (SPM). The BoF method is nothing but the presenting an image in the form of a histogram of its local features which are obtained by extraction. First, feature points are detected located on the input image of iris, and then descriptors like "SIFT (Scale Invariant Feedback Transform) " is extracted from each feature point which are located on the iris image. This results in the "Descriptor" layer formation. After that, a codebook with $\mathrm{M}$ entries into it is applied to quantize each descriptor and generate the "Code" layer which is next to descriptor layer, where each descriptor is converted into an $\mathrm{R}^{\mathrm{M}}$ code. If hard vector quantization (VQ) is used for the quantization, each code has only one non-zero element which satisfies criteria, while for soft-VQ, a group of elements can be non-zero. Then in the "SPM ( Spatial Pyramis Matching)" layer, multiple codes from inside of the each sub-region are pooled together by averaging method and normalizing into a histogram. Result, the histograms from all sub-regions are concatenated together to generate the final representation of the iris image for classification. The use of novel and practical coding scheme called Locality-constrained Linear Coding (LLC), which can be seem as a effective and fast implementation of LCC that utilizes the locality constraint to project each descriptor into its local-coordinate system. 


\section{LITERATURE SURVEY}

In this section we discussed about literature survey on iris classification and recognition.

In [1], Zhenan Sun, Hui Zhang, Tieniu Tan, Jianyu Wang, et al. This paper proposes framework for iris image classification based on texture analysis and extracting the features. A texture pattern representation method called Hierarchical Visual Codebook (HVC) is proposed to encode the texture primitives of iris images. The proposed HVC method is an combination of existing Bag-of-Words models, namely Vocabulary Tree (VT), and Locality-constrained Linear Coding (LLC). The HVC adopts a coarse-to-fine visual coding strategy and takes advantages of both VT and LLC for accurate and sparse representation of iris texture. Experimental results demonstrate that the proposed iris image classification method achieves state-of-the-art performance for iris liveness detection, race classification, and coarse-tofine iris identification.

In [2], Alghamdi, et al. the biometric data is converted into the binary stream which in result then divided into the block of size 128. After that the one block is selected randomly and used as a encryption key but before using it as a encryption key firstly it is encrypted using the quantum algorithms. Then this encrypted key is used to encrypt the other blocks. The decryption process is reverse of this.

In [3], M. Sunder et al. to recognize the individual's identity many system use the global and the local texture pattern. This paper uses the macro-features of the anterior surface of the RGB images for getting the matching features. And this feature (macro) includes the moles, melanoma etc. The main aim will be to retrieve the matching iris image corresponding to the given macro-feature from the database. To solve this problem author has used the SIFT i.e Scale-Invariant Feature Transform for getting the macro-features. The experiments are done on the subset of 770 distinct irides from Miles Research Iris Database and the results suggests that there is possibility of getting macro-features for matching and retrieval.

In [4], Z. Sun et. al. human iris contains abundant information about its identity which can therefore be used for the person's identity. The problem is how to use this information for getting features which in this case are represented as textural information. The author here proposes to use the ordinal measures for the representation of the features. The aim is to characterize the relationship (qualitative) between iris regions instead of the measuring iris image structures. This kind of representation may result in loss of image-specific information, but it achieves the good trade-off between robustness and distinctiveness. This paper proves that ordinal measures are the intrinsic features of iris patterns and invariant to changes in illumination. The computational complexity of the ordinal measures is low. Therefore it is used for highly efficient iris recognition system. Ordinal measures are generally used for the image analysis. They have showed the effective experimental results on three public databases. In this paper, they develop multi-lobe differential filters for the computation of ordinal measures with flexible intra-lobe and inter-lobe parameters such as location, scale, orientation, and distance.

In [5], E. Lee et al. have proposed the fake iris detection system and the input for the system is the iris image. They trying to detect the fake iris image with the help of Purkinje image which includes the front and back surface of the cornea, and the front and back surface of the lens. Here, the four reflected images of incident light on each optical surface are mentioned as Purkinje images. The theoretical positions and the distances between Purkinje images are calculated because they are used to get the information about the fake iris. The experimental results are quite impressive i.e FRR(False Rejection Rate of rejecting live iris as fake one) was 0.33 and FAR (False Acceptance Rate for accepting fake iris as live one) was $0.33 \%$.

In [6], X. He et al. the fake iris image detection is done to solve the problem of the authentication of the person's identity which is the severe issue now-a-days. This paper proposes the use of 2-D Fourier spectra along with iris image quality assessment. Two steps are considered into this fake iris detection method. Firstly, noise removal technique i.e quality assessment is done which helps to remove the defocused and motion blurred fake iris. Secondly, Fourier spectra statistical properties are applied to clear the fake iris image detection. The experimental results are pretty good to detect the printed and the photo iris images efficiently.

In [7], Z. Wei et al. the liveness detection problem is discussed which includes the fake and real iris image detection i.e counterfeit iris detection. The attack on recognition system here considered is colored contact lens which having texture pattern printed on it and this is wear by the intruder trying to fraud the authentication system. The three measures are considered over here measuring iris edge sharpness, applying Iris-Texton feature for characterizing the visual primitives of iris textures and using selected features based on co-occurrence matrix (CM). For experimental reasons they have used two databases which contains the 640 fake iris images. The proposed system is concern about the shape of the iris mainly which is inspected by the three methods mentioned.

The literature survey of iris image classification is weaker than that of the iris image recognition.

\section{PROPOSED APPROACH FRAMEWORK AND DESIGN \\ 3.1 Problem Definition}

Pattern recognition is the mutual issue with the iris image classification and the recognition i.e. classification of iris pictures into some predefined categories. The sole distinction is that the definition of sophistication labels at macro or small scale. For recognition, the category label is the identity of an individual (individual identity). In classification, the category label could correspond to a gaggle of subjects with similar properties of iris pictures (group identity). So the solution of iris image classification is considerably totally different to iris recognition. Iris texture naturally has distinctive pattern for every subject therefore extraction of the severally specific options are there $\{$ to distinguish |to totally differentiate |to tell apart\} for different subjects. However, iris image classification has to realize the stable relationship of similar iris texture options between totally different subjects. Such an inter-person relationship is also outlined manually without support of solid physiological evidences. Therefore, iris image classification is really a more complex issue compared with iris recognition. 


\subsection{System Architecture}

The proposed system uses the bio-chaotic algorithm which helps to encrypt the iris images and store them into the database. Here iris image is used in the form of binary pattern then this binary data is divided into the block. One block is chosen randomly to use as the secrete encryption key. This key in return also encrypted using quantum cryptographic algorithm. Now this encrypted secrete key is used to encrypt the other blocks. For decryption reverse process is applied.

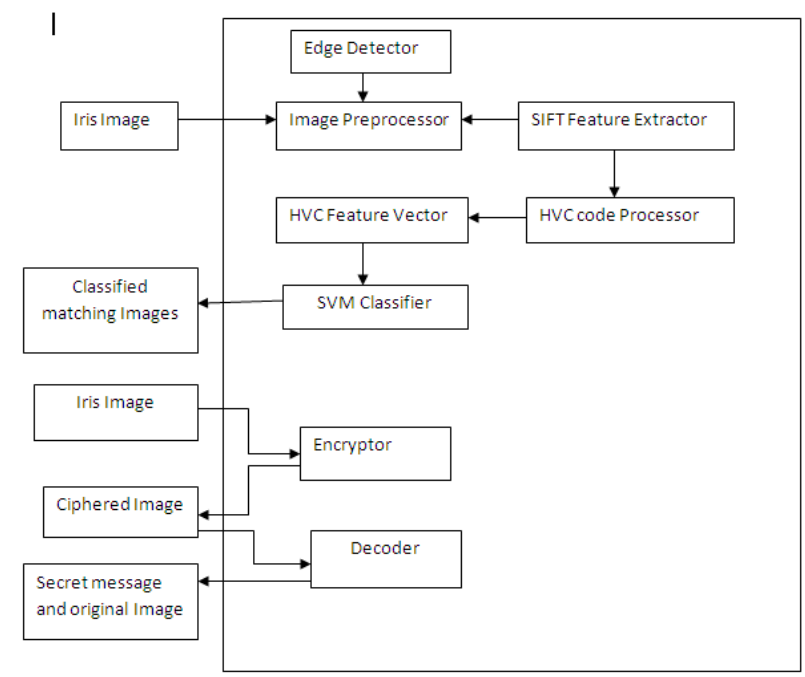

Fig 1: System Architecture

\subsubsection{Mathematical Model}

\section{Set Theory:}

Use set theory, and/or relevant mathematics to model the situation you are facing in your research.

The set theory using in this are as follow: $\mathrm{S}=\{\mathrm{U}, \mathrm{I}, \mathrm{C}\}$

Let $\mathrm{I}=\{\mathrm{U} 1, \mathrm{U} 2, \ldots, \mathrm{Un}\}$ which is set of end users.

Let $U 1=\{$ uf1, uf2, uf $3, \ldots$, ufn $\}$ which is the set of input iris image features.

Let $I$ is the set of ground truth IRIS images

$\mathrm{I}=\{\mathrm{x} 1, \mathrm{x} 2, \mathrm{x} 3, \mathrm{x} 4, \mathrm{x} 5, \mathrm{x} 6, \mathrm{x} 7\}$

Let FI $=\{\mathrm{f} 1, \mathrm{f} 2, \mathrm{f} 3 \ldots \ldots, \mathrm{fn}\}$ Set for fake iris images.

Now let us consider a set of ground truth images for each feature extracted from iris image.

For iris image $x 1=\{x f 1, x f 2, x f 3, \ldots, x f n\}$ are the feature set. For iris image $\mathrm{x} 2=\{\mathrm{xf1}, \mathrm{xf} 2, \mathrm{xf} 3, \ldots, \mathrm{xfn}\}$ are the components. This will apply to rest of iris images.

The input iris image features are compared against the real and fake iris features and according to that image is classified into particular category.

Let $\mathrm{C}$ be the recognition output for each image store into particular category .

$$
\mathrm{C}=\{\mathrm{C} 1, \mathrm{C} 2, \mathrm{C} 3, \mathrm{C} 4, . ., \mathrm{Cn}\}
$$

\section{Bio-Chaotic Algorithm:}

For iris image Encryption the use of Bio-chaotic algorithm is a good idea. The steps to follow are given below:

Initial Condition $=2^{n}-1 ; \mathrm{n}=1,2,3$..so on.

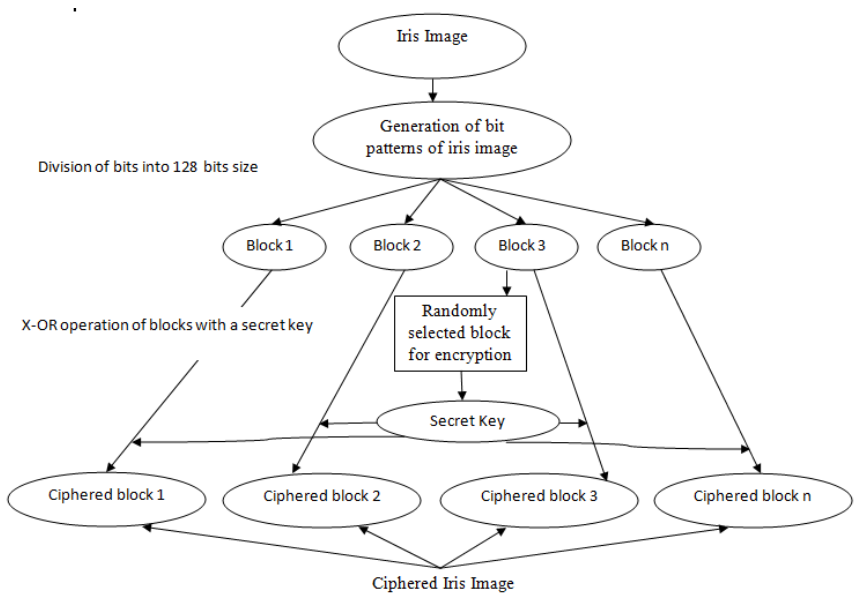

Fig 2: Block Diagram of Bio-Chaotic Algorithm

This initial condition is used to create secret key by using the LFSR method. An LFSR of length $n$ over a finite field $P_{q}$ consist of $n$ stages $\left(a_{n-1}, a_{n-2}, a_{n-3}, \ldots \ldots, a_{0}\right)$ with ai $\epsilon$ of $P q$, and a polynomial.

$B(x)=1+c_{1} x_{1}+c_{2} x_{2}+\ldots .+c_{n} x_{n}$ over $\mathrm{P}_{\mathrm{q}}$

The X-oring of the secret key and iris template simultaneously

to generate the biometric key by using the equation,

Biometric key $=a_{1}$ xor $b_{1}, a_{2}$ xor $b_{2}, \ldots, a_{n}$ xor $b_{n}$

Biometric key is then Xored with other blocks of the iris template (divided into blocks of 128 bits/block) which encrypts the image in a way that no intruder or attacker can easily decrypt the image which is the main advantage of this algorithm.

To make the this algorithm more stronger and secure addition of the chaotic function to the biometric key will be beneficial and apply it over the iris image to encrypt it in a more secure fashion.

The decryption process is reverse in which the used image is carried on by the same fashion using the same key used for the encryption process but in the opposite direction

Plain image $=$ Ciphered image $x$-or key

\section{WORK DONE}

In this section discussion of the practical environment, scenarios, performance metrics is provided.

\subsection{Input}

In this iris image is the input for our practical experiment belongs to online databases like CASIA, ND-Contact.

\subsection{Image Preprocessing and Classification}

The normalization of iris image is done. The Gaussian blur is used to remove the noise from the image for detecting the features of the iris image. The normalized image obtained after preprocessing technique which can be further be used for feature extraction. 
Canny edge detection technique is use to extract the edges. Feature points are detected or densely located on the input image, and descriptors such as "SIFT" or "color moment" are extracted from each feature point. Then the clustering of these extracted features is done the closely related features will be grouped into the same cluster. After that pooling is done using SPM to acquire the HVC feature vector. Depending upon that SVM classifier works on classifying the image into fake or genuine. For securing these iris templates BCA is used which is nothing but the encrypting image by partitioning it and secrete key.

\subsection{Output}

The histogram will be generate of the encrypted as well as original image which shows that little bit change into the image by hacker can changed slightly (for example, flipping a single bit) the output changes significantly.

\subsection{Results}

The results compared here are time graph between existing and proposed system is shown in below figure.

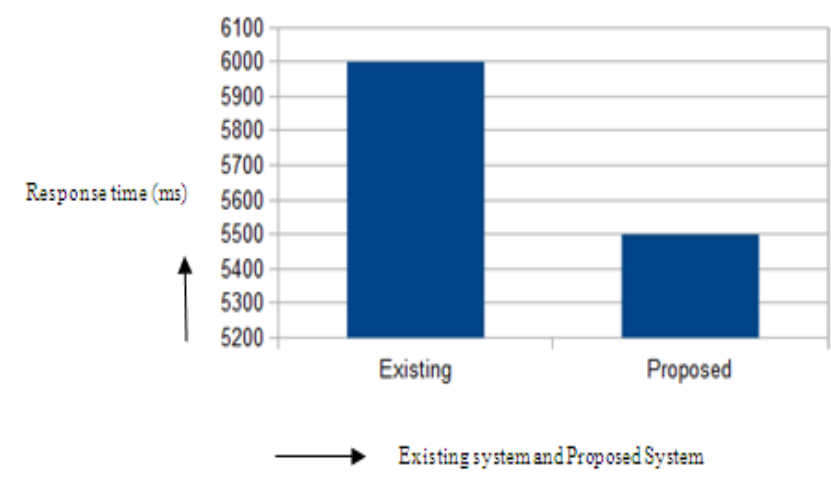

Fig 3: Time Comparison Graph

$\mathrm{X}$-axis: Existing system and Proposed System

Y-axis: Response Time (ms)

The existing system and proposed system against response time is plotted. The response time is in milliseconds. The time complexity of the proposed system is far less than the existing system.

Figure 3 shows the precision graph between existing system and proposed system

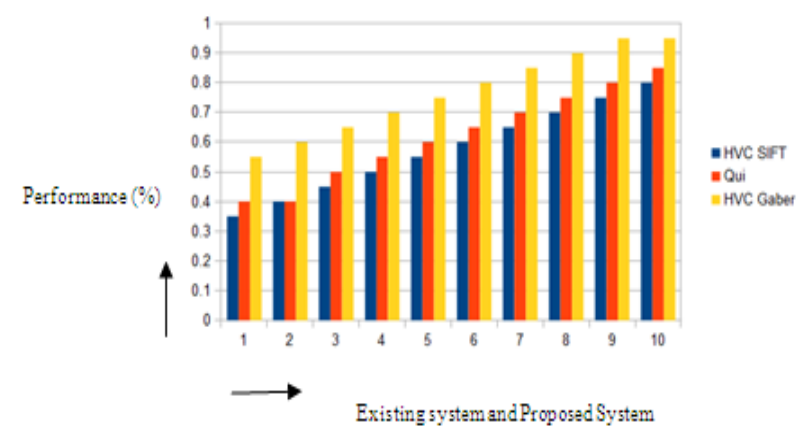

X-axis: Existing system and Proposed System Y-axis: Performance

Most of the evaluations of vocabulary characteristics were based on image retrieval performance. The result of the query of the image retrieval system is a ranked list of images. It is desirable to consider the order in which the returned images are presented. Average Precision (AP) represents the area under Precision-Recall curve or a query. Precision and Recall are defined below:

\section{Precision $=$ retrieved relevant images/retrieved images} Recall $=$ retrieved relevant images/all relevant images

The performance graph is plotted, the hierarchical visual codebook along with scale invariant feature transform, Qui, hierarchical visual codebook along with Gabor are at $\mathrm{x}$-axis and the performance at $y$-axis.

Below are the screenshots of the our working system:

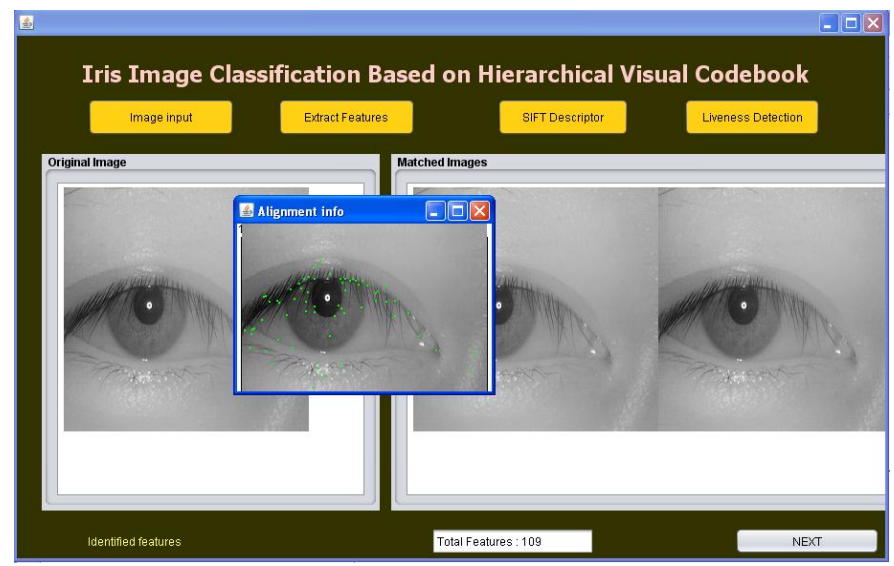

Above is the homepage of the system where image features are extracted and localize. The image matching algorithm is used over here and can set the percentage of features matches so that can get matching images to the input image.

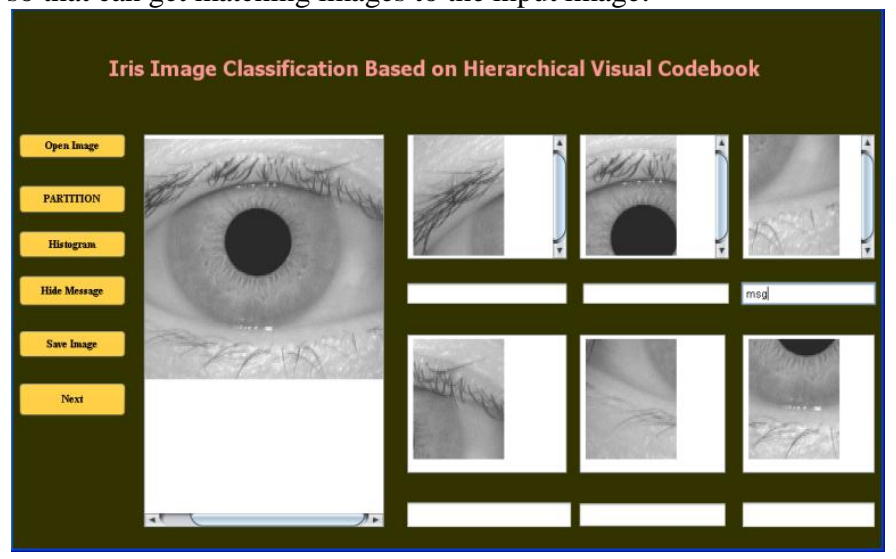

In above snapshot the iris input image is divided into the blocks so that secret message can be hide into the one of the random block and attacker would not be able to guess it.

Fig 4: Performance Graph 


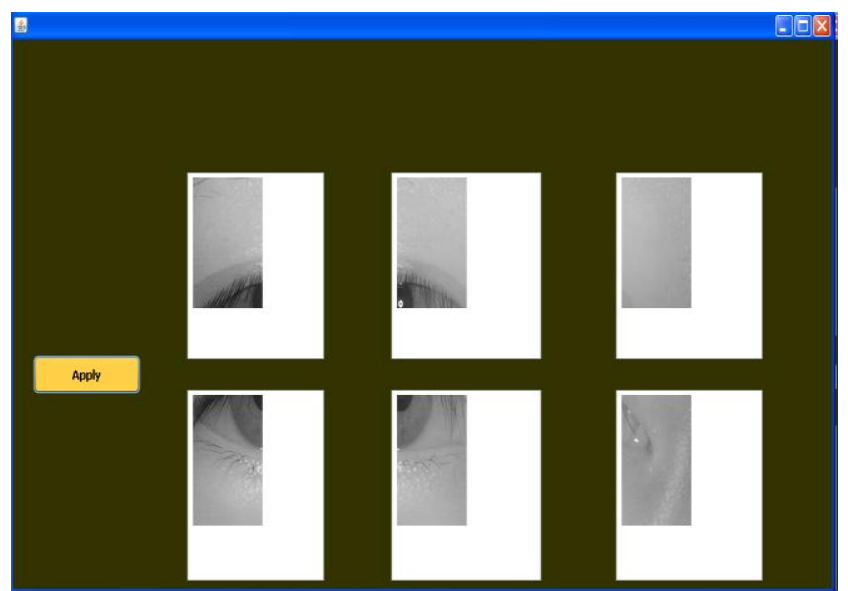

Then histogram is generated of the original image after clicking on apply button.

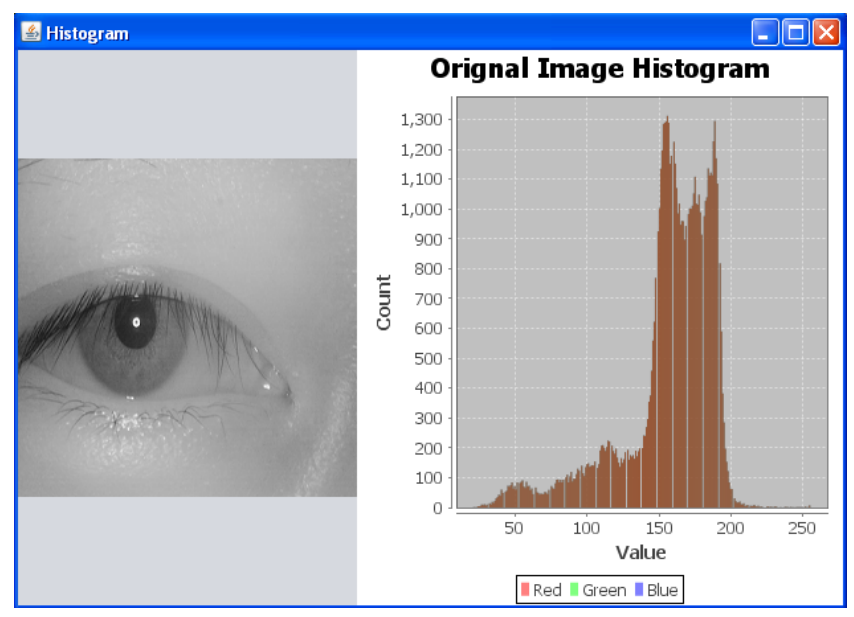

The histogram of the original image will be look like above image.

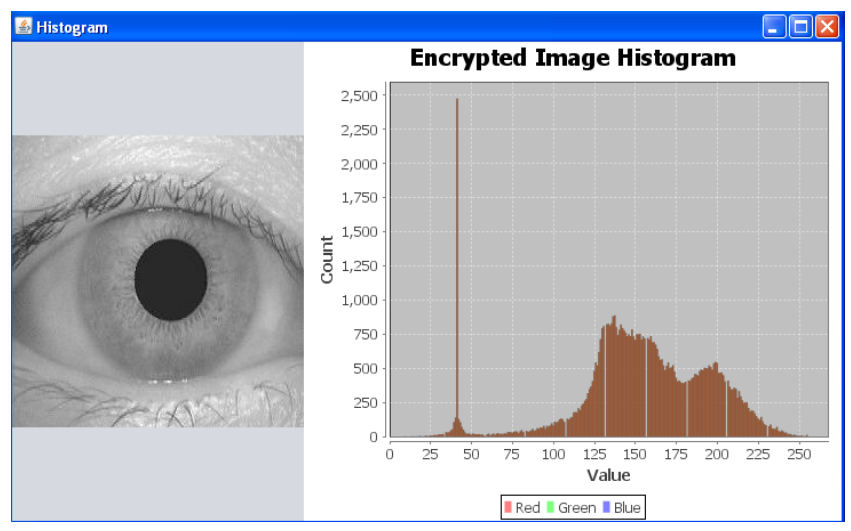

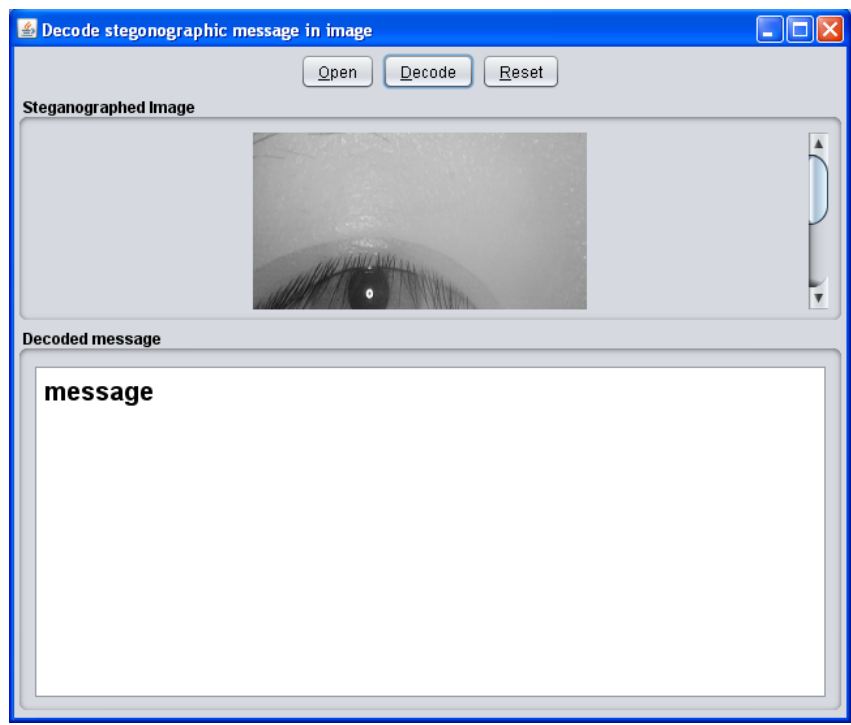

\section{CONCLUSION}

Iris image classification includes the clustering of the iris images which shares the similar features. So, use of this classification for detecting fake and real iris images. This helps to group the iris images into different categories. The iris images sharing same features will get clustered under similar category. So the computational price of feature extraction and matching for multiple iris image classification tasks is greatly reduced. Along with the classification there is a addition of the bio-chaotic algorithm to encrypt the images and store them into the database which results into the efficient security system using hierarchical visual codebook and bio-chaotic algorithm. At the receiver end receiver will get the encrypted image via e-mail system which is sent by the sender from his system. After that receiver will decode the image using secret key and generate original image as well as message hidden into it. For future the system can be extended to use on any image type rather than on iris image.

\section{ACKNOWLEDGMENT}

I would like to take opportunity to acknowledge the contribution of certain people without which it would not have been possible to complete this paper work. I would like to express my special thanks to my guide Prof. Deeksha Bhardwaj for her valuable guidance and support.

\section{REFERENCES}

[1] Sun, Zheng, et. al. Iris Image Classification Based on Hierarchical Visual Codebook (2014):1-1.

[2] Alg hamdi, Abdullah Sharaf, et al. "Bio-chaotic stream cipher -based iris image encryption." Computational science and engineering, 2009. CSE'09. International conference on. Vol.2. IEEE, 2009

[3] M. Sunder and A. Ross, "Iris image retrieval based on macro-features," in Proc. ICPR, Istanbul, Turkey, 2010, pp. 1318-1321.

[4] Z. Sun and T. Tan, "Ordinal measures for iris recognition," IEEE Trans. Pattern Anal. Mach. Intell., vol. 31, no. 12, pp. 2211-2226, Dec. 2009

[5] E. Lee, K. Park, and J. Kim, "Fake iris detection by using purkinje image," in Proc. ICB, Hong Kong, China, 2006, pp. 397-403. 
[6] X. He, Y. Lu, and P. Shi, "A fake iris detection method based on FFT and quality assessment," inProc. Chinese Conf. Pattern Recognition, Beijing, China, 2008, pp. 316-319.

[7] Z. Wei, X. Qiu, Z. Sun, and T. Tan, "Counterfeit iris detection based on texture analysis," inProc. ICPR, Tampa, FL, USA, 2008, pp. 1-4.

[8] Z. He, Z. Sun, T. Tan, and Z. Wei, "Efficient iris spoof detection via boosted local binary patterns," inProc. ICB, Alghero, Italy, 2009, pp. 1080-1090.

[9] H. Zhang, Z. Sun, and T. Tan, "Contact lens detection based on weighted LBP," in Proc. ICPR, Istanbul, Turkey, 2010, pp. 4279-4282.

[10] S. Gutta, H. Wechsler, and P. Phillips, "Gender and ethnic classification of face images," inProc. FG, Nara, Japan, 1998, pp. 194-199.

[11] G. Shakhnarovich, P. Viola, and B. Moghaddam, "A unified learning framework for real time face detection and classification," in Proc. FG, Washington, DC, USA, 2002, pp. 14-21.
[12] X. Lu and A. Jain, "Ethnicity identification from face images," in Proc. SPIE Defense and Security Symp., vol. 5404. 2004, pp. 114-123.

[13] X. Qiu, Z. Sun, and T. Tan, "Global texture analysis of iris images for ethnic classification," in Proc. ICB, Hong Kong, China, 2006, pp. 411-418.

[14] X. Qiu, Z. Sun, and T. Tan, "Learning appearance primitives of iris images for ethnic classification," inProc. ICIP,vol.2.SanAntonio, TX, USA, 2007, pp. 405-408.

[15] H. Zhang, Z. Sun, T. Tan, and J. Wang, "Ethnic classification based on iris images," inProc. Chinese Conf. Biometric Recognition, Beijing, China, 2011, pp. 82-90.

[16] J. Lyle, P. Miller, S. Pundlik, and D. Woodard, "Soft biometric classification using periocular region features," in Proc. BTAS, Washington, DC, USA, 2010, pp. 1-7.

[17] L. Yu, D. Zhang, K. Wang, and W. Yang, "Coarse iris classification using box-counting to estimate fractal dimensions," Pattern Recognit., vol. 38, no. 11, pp. 1791-1798, 2005. 